\title{
Epithelial tethering of MUC5AC-rich mucus impairs mucociliary transport in asthma
}

\author{
Luke R. Bonser, ${ }^{1}$ Lorna Zlock,, ${ }^{2}$ Walter Finkbeiner, ${ }^{2}$ and David J. Erle ${ }^{1}$ \\ 'Lung Biology Center and 2Department of Pathology, UCSF, San Francisco, California, USA.
}

\begin{abstract}
The development of pathologic mucus, which is not readily cleared from the airways, is an important contributor to the morbidity and mortality associated with asthma. It is not clear how the major airway mucins MUC5AC and MUC5B are organized within the mucus gel or how this gel contributes to airway obstruction in asthma. Here, we demonstrated that mucus plugs from individuals with fatal asthma are heterogeneous gels with distinct MUC5AC- and MUC5B-containing domains. Stimulation of cultured human bronchial epithelial cells with IL-13, a key mediator in asthma, induced the formation of heterogeneous mucus gels and dramatically impaired mucociliary transport. Impaired transport was not associated with defects in ciliary function but instead was related to tethering of MUC5AC-containing mucus gel domains to mucus-producing cells in the epithelium. Replacement of tethered mucus with untethered mucus restored mucociliary transport. Together, our results indicate that tethering of MUC5AC-containing domains to the epithelium causes mucostasis and likely represents a major cause of mucus plugging in asthma.
\end{abstract}

\section{Introduction}

Asthma affects approximately 300 million people worldwide and causes approximately 250,000 deaths annually (1). Tracheobronchial mucociliary clearance is impaired in stable asthma (2) and worsens during acute exacerbations (3). The principal cause of death from asthma is asphyxiation from intraluminal airway obstruction by widespread mucus plugs (4-6). In other diseases, mucus transport is impaired due to the absence or immotility of cilia (7), as seen in primary ciliary dyskinesia (PCD), or to periciliary liquid depletion (8) and impaired mucus detachment from submucosal glands (9), as seen in cystic fibrosis (CF). However, the cause of impaired mucus transport in asthma is poorly understood.

The viscoelastic properties of airway mucus depend on 2 gelforming mucin glycoproteins, MUC5B and MUC5AC (10). MUC5B is produced in submucosal glands and by secretory cells within the airway epithelium (11). MUC5B-deficient mice have impaired mucociliary clearance, leading to pulmonary infections (12). MUC5AC is produced by specialized airway epithelial cells known as mucous (or goblet) cells (11). MUC5AC-deficient mice have normal mucociliary transport and are protected from mucus plugging in an allergic asthma model $(12,13)$. The type 2 cytokine IL- 13 is necessary (14) and sufficient (15) for mucus induction in mouse asthma models. Many individuals with asthma, especially those with high levels of IL-13, have increased MUC5AC mRNA levels but decreased MUC5B mRNA levels (16). Increases in MUC5AC and MUC5B protein concentrations have been reported in sputum from individuals with asthma (17) and in a mucus plug in an individual with fatal asthma (18). We sought to determine whether changes in mucus composition, organization, and function contribute to impaired mucus transport in asthma.

Conflict of interest: The authors have declared that no conflict of interest exists. Submitted: October 2, 2015; Accepted: March 22, 2016.

Reference information: J Clin Invest. 2016;126(6):2367-2371. doi:10.1172/JCI84910.

\section{Results and Discussion}

MUC5AC and MUC5B localize to distinct domains within mucus plugs in fatal asthma. We compared mucin staining in airway sections from controls and individuals with fatal asthma. In 4 controls, we detected airway epithelial cells containing MUC5AC, MUC5B, or both mucins (Supplemental Figure 1A; supplemental material available online with this article; doi:10.1172/JCI84910DS1) but found no intraluminal mucus (Figure 1, A and B), presumably because intraluminal mucus was removed during standard aqueous-based fixation. In the fatal asthma samples, we detected more MUC5AC- but fewer MUC5B-containing cells (Figure 1, C and D, and Supplemental Figure 1). Large intraluminal mucus plugs were evident in 4 of 7 individuals with fatal asthma (Figure 1, C and D). Mucin staining within the mucus plugs was heterogeneous: $64 \% \pm$ $7 \%$ (mean \pm SEM) of the cross-sectional area of the plugs contained MUC5AC but no detectable MUC5B; 25\% $\pm 5 \%$ contained MUC5B but no MUC5AC; and only $11 \% \pm 2 \%$ stained for both MUC5AC and MUC5B. MUC5AC and MUC5B therefore localize predominantly to distinct domains within mucus plugs in fatal asthma.

IL-13 induces a heterogeneous mucus gel in vitro. IL-13 is a major driver of asthma in a large subset of individuals and potently induces MUC5AC expression (16). We investigated whether IL-13 stimulation was sufficient to cause changes in mucus gel composition and organization similar to those seen in fatal asthma. IL-13 stimulation of cultured human bronchial epithelial (HBE) cells from multiple individuals consistently increased MUC5AC expression, whereas MUC5B expression was decreased or unaffected (Supplemental Figure 2). We detected numerous MUC5B-containing cells in unstimulated and IL-13-stimulated cultures and a large increase in MUC5AC-containing cells after IL-13 stimulation (Supplemental Figure 3). In rarer cells staining for both mucins, MUC5AC and MUC5B localized to different granules, suggesting discrete secretory pathways and consistent with findings in mice (19). 

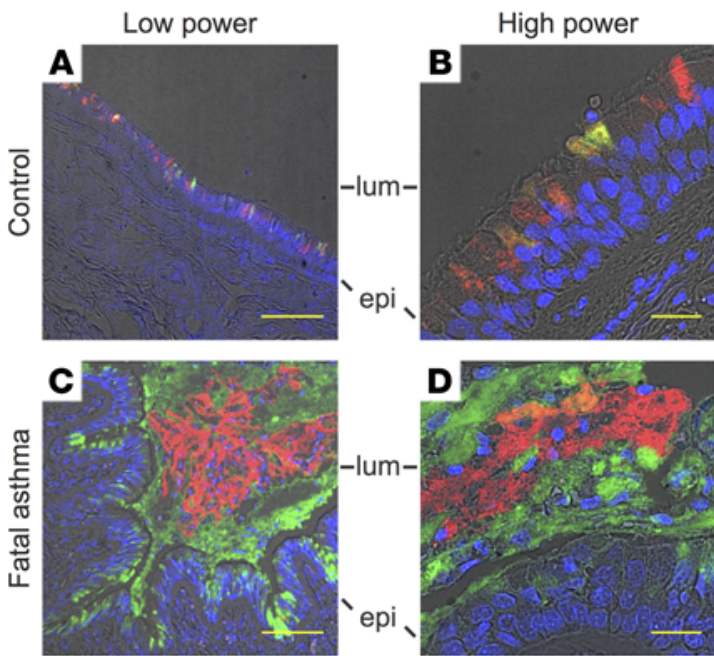
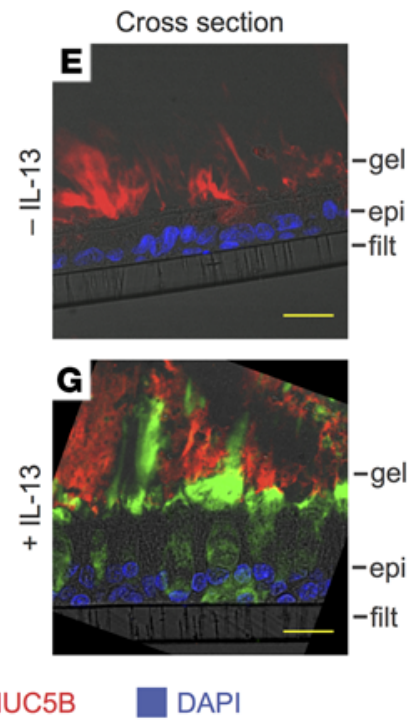

DAPI
Gel (whole-mount)
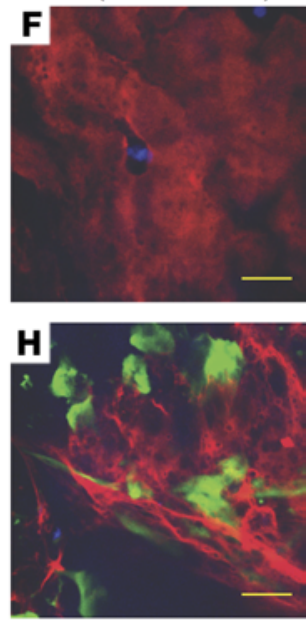

MUC5AC

MUC5B

Figure 1. Distinct MUC5AC- and MUC5B-rich domains in mucus from fatal asthma and IL-13-stimulated HBE cells. (A-D) Immunohistochemical staining of mucins in airways from controls $(n=4)$ and individuals with fatal asthma $(n=7)$. (E-H) Immunohistochemical staining of sections and whole-mount preparations from 3 unstimulated (- IL-13) or 3 IL-13-stimulated (+ IL-13) HBE cultures. Whole-mount images represent optical sections through the gel (parallel to the plane of the epithelium). epi, epithelium; lum, airway lumen; gel, extracellular mucus gel; filt, filter support. Scale bars: $100 \mu \mathrm{m}$ (A and C) and $20 \mu \mathrm{m}$ (B and D-H).

To explore how MUC5AC and MUC5B were organized within extracellular mucus gels, we cultured HBE cells from 3 donors in the absence or presence of IL-13, allowed mucus to accumulate for 3 days, processed the cultures with a nonaqueous fixative, and stained for mucins (2-3 sections, 1 whole-mount preparation per donor). A MUC5B-containing gel was evident in all unstimulated cultures (Figure 1, E and F). MUC5AC staining was not observed but was occasionally seen in other experiments. In contrast, all IL-13-stimulated cultures showed prominent MUC5AC staining and some MUC5B staining (Figure 1, G and H). Gels from IL-13stimulated cultures were heterogeneous: $59 \% \pm 8 \%$ of the crosssectional area of gels from IL-13-stimulated cultures contained MUC5AC but no detectable MUC5B; $35 \% \pm 8 \%$ contained MUC5B but no MUC5AC; and $6 \% \pm 1 \%$ stained for both MUC5AC and MUC5B. Hence, IL-13 treatment produced heterogeneous mucus gels with distinct MUC5AC- and MUC5B-containing domains that were similar to those seen in fatal asthma.

IL-13 impairs mucus transport. We determined whether IL-13induced alterations in mucin composition and organization were associated with changes in the transport properties of the mucus gel by tracking fluorescent microspheres deposited on the gel surface (Figure 2, A and B). Microsphere transport was markedly reduced by IL-13 stimulation (median speed decreased by 21- to 238-fold, Figure 2C). Hence, IL-13 is sufficient to severely impair mucus transport by airway epithelial cells.

Ciliary beating and the periciliary layer are preserved despite impaired mucociliary transport. Ciliary beating propels the mucus gel. The absence of functional multiciliated cells, as seen in PCD (7), or the collapse of the periciliary layer, as seen in CF models (8), leads to impaired mucus gel transport. The IL-13 stimulation conditions we used did not prevent the differentiation of multiciliated cells (Supplemental Figure 4, A and B). Ciliary beat frequency was unaffected by IL-13 (Figure 2D). A previous study showed that marked increases in mucus gel solids lead to pronounced periciliary liquid depletion and ciliary collapse (8). The mean concentration of mucus solids from our unstimulated cultures was $1.5 \%$ (Supplemental Figure 4C), similar to the $1.7 \%$ reported in normal human sputum (20). Mucus solids increased to $2.7 \%$ after IL-13 stimulation but remained well below the approximately $7 \%$ reported to collapse the periciliary layer and substantially impair mucociliary transport (8). Measurements of ciliary length confirmed that IL-13 stimulation did not cause ciliary collapse (Supplemental Figure 4D). Impaired mucus gel transport in our model system is therefore not explained by loss of multiciliated cells, changes in ciliary beating, or collapse of the periciliary layer.

MUC5AC-containing domains of mucus are tethered to the airway epithelium. We next addressed whether changes in the mucus gel accounted for the impaired transport. In a piglet CF model, loss of the cystic fibrosis transmembrane conductance regulator (CFTR) results in more adherent submucosal gland mucus that remains tethered to gland duct openings, leading to impaired mucociliary transport (9). The pathogenesis of CF is fundamentally different from that of asthma, and the defective mucus transport observed in IL-13-stimulated HBE cells occurred in the absence of submucosal glands. Furthermore, submucosal glands in healthy individuals and individuals with $\mathrm{CF}$ produce abundant amounts of MUC5B but no detectable MUC5AC (11), whereas mucus from fatal asthma mucus plugs or IL-13-stimulated HBE cells contained high MUC5AC levels and relatively low MUC5B levels (see above). We hypothesized that MUC5AC-containing mucin domains are tethered to the airway epithelium.

We began by examining the relationship of gel mucins to the epithelium. The peak intensity of MUC5AC staining $(7.6 \pm 1.4 \mu \mathrm{m}$ above the epithelial surface, 3 image stacks from each of 3 donors) was closer to the epithelium than the peak of MUC5B staining $(11.7 \pm 1.2 \mu \mathrm{m}$; $P<0.0001$, by Mann-Whitney $U$ test, Figure 3A). There was extensive 

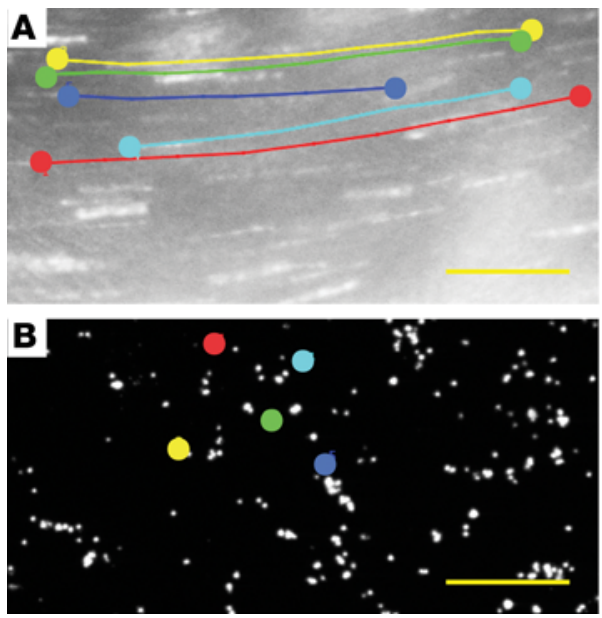

C

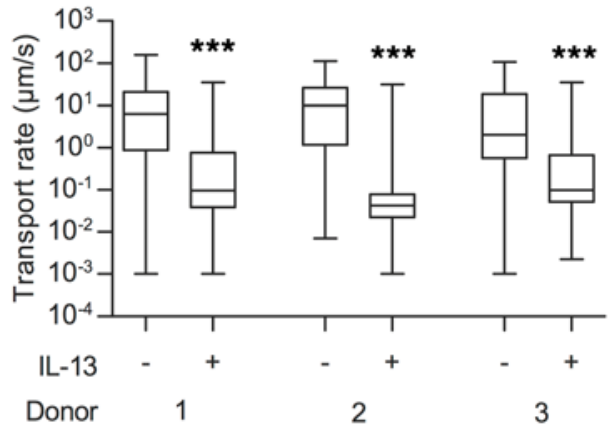

D

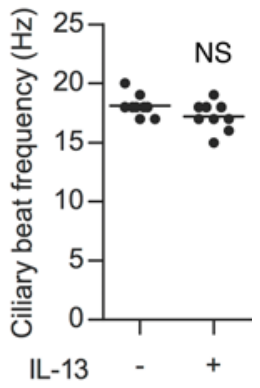

Figure 2. IL-13 impairs mucus transport. (A and B) Paths of fluorescent microspheres deposited on gels from unstimulated (-) and IL-13-stimulated (+) HBE cell cultures. The final image from a 10-second image sequence is shown. The trajectories of 5 representative microspheres are indicated by the colored dots. Particles from the unstimulated culture (A) had similar trajectories, whereas particles from the IL-13-stimulated culture had minimal movement (B). Scale bars: $20 \mu \mathrm{m}$. (C) Distribution of microsphere velocities for unstimulated and IL-13-stimulated cultures from 3 individuals (donors 1-3). For both unstimulated and IL-13-stimulated cultures, velocities were determined from 810 to 1,198 microspheres from 3 videos; these microspheres were obtained from triplicate cultures from each of 3 individuals. ${ }^{* *} P<0.0001$ compared with unstimulated cultures, by Mann-Whitney $U$ test. Boxes extend from the 25th to the 75th percentile, the line within the box indicates the median, and the whiskers represent minimum and maximum values. The boxes extend from the 25th to 75th percentiles, the line within the box indicates the median, and the whiskers represent minimum and maximum values. (D) Mean ciliary beat frequency was determined from 3 fields; cultures were from 3 individuals. NS compared with unstimulated cultures, by Student's $t$ test.

contact between the apical surface of mucous cells and MUC5ACcontaining areas of the extracellular gel (Figure 3, B-D, and Supplemental Figure 5). MUC5AC-containing regions of the gel frequently traversed the approximately $6-\mu \mathrm{m}$ region above the epithelium corresponding to the periciliary layer (Figure 3, A and C). This region was depleted of MUC5B, and MUC5B-containing regions were rarely contiguous with a MUC5B-containing cell $(4.7 \% \pm 3.4 \%)$. In contrast, MUC5AC-containing regions were frequently continuous with MUC5AC-containing mucous cells $(59.4 \% \pm 5.7 \% ; P<0.01$, by Student's $t$ test). Hence, MUC5AC-containing domains were frequently found in continuity with MUC5AC-producing mucous cells, whereas MUC5B-containing domains were not closely associated with epithelial cells. Similarly, regions of fatal asthma mucus plugs close to the airway epithelium predominantly contained MUC5AC and not MUC5B (Figure 1, C and D, and Supplemental Figure 6).

We directly examined whether MUC5AC-containing domains of the mucus gel were tethered to the epithelial surface by assessing the effects of washing the apical surfaces of HBE cell cultures from 3 donors (3 sections per donor). Unwashed, unstimulated cultures had MUC5B-containing gels (9 of 9 sections, Figure 3E); these gels were not tethered, since no gels were seen after washing unstimulated cultures ( 0 of 9 sections, Figure 3F). Unwashed, IL-13-stimulated cultures had heterogeneous gels containing distinct MUC5AC-dominant and MUC5B-dominant domains (9 of 9 sections, Figure $3 G$ ). After washing, extracellular mucus remained attached to the epithelium (8 of 9 sections, Figure 3H). MUC5AC was present in all 8 washed sections with mucus, but MUC5B was evident in only 3 sections. The ratio of MUC5AC/MUC5B-stained area increased from $2.0 \pm 0.3$ in unwashed cultures to $6.0 \pm 1.1$ after washing $(P<0.01$, by Student's $t$ test), indicating preferential attachment of MUC5AC-containing regions. The MUC5B-containing regions seen after washing of IL-13-stimulated cultures were typically associated with MUC5AC-containing regions and were not in direct contact with the epithelium. Hence, MUC5ACcontaining regions found in abnormal airway mucus gels are tethered to the epithelium.

Replacement of tethered gels restores mucociliary transport. Mucins are cross-linked by intermolecular disulfide bonds, and addition of the reducing agent DTT to the wash solution led to removal of detectable mucins from IL-13-stimulated as well as unstimulated cultures (Supplemental Figure 7). We analyzed transport in unstimulated and IL-13-stimulated cultures before washing, after washing with DTT, and, finally, after replacement of the gel with a transplanted gel produced by unstimulated donor cells (Figure 3I). Consistent with our previous results, IL-13 markedly reduced the velocity of microspheres prior to removal of the gel. It was recently reported that removal of the mucus gel from healthy airways did not abolish particle transport but instead modestly increased transport velocity (21). We obtained similar results after removal of gels from unstimulated cultures. In comparison, removal of tethered gels from IL-13stimulated cultures dramatically increased transport to levels that were only modestly lower than those seen in unstimulated cultures. Finally, replacement of the gel with mucus from unstimulated cultures almost normalized transport velocity on IL-13-stimulated cells. These data indicate that mucus transport is restored by replacement of epithelium-tethered MUC5AC-rich gels with untethered gels, demonstrating a mechanism for impaired mucociliary transport that likely plays a significant role in severe asthma.

Mucus hypersecretion is a prominent feature of asthma (22), and this work provides what we believe to be new insights into how changes in mucus contribute to airway obstruction in asthma. An earlier autopsy study demonstrated a large increase in the frequency of airway mucous cells in continuity with intraluminal mucus in individuals with asthma (23). 

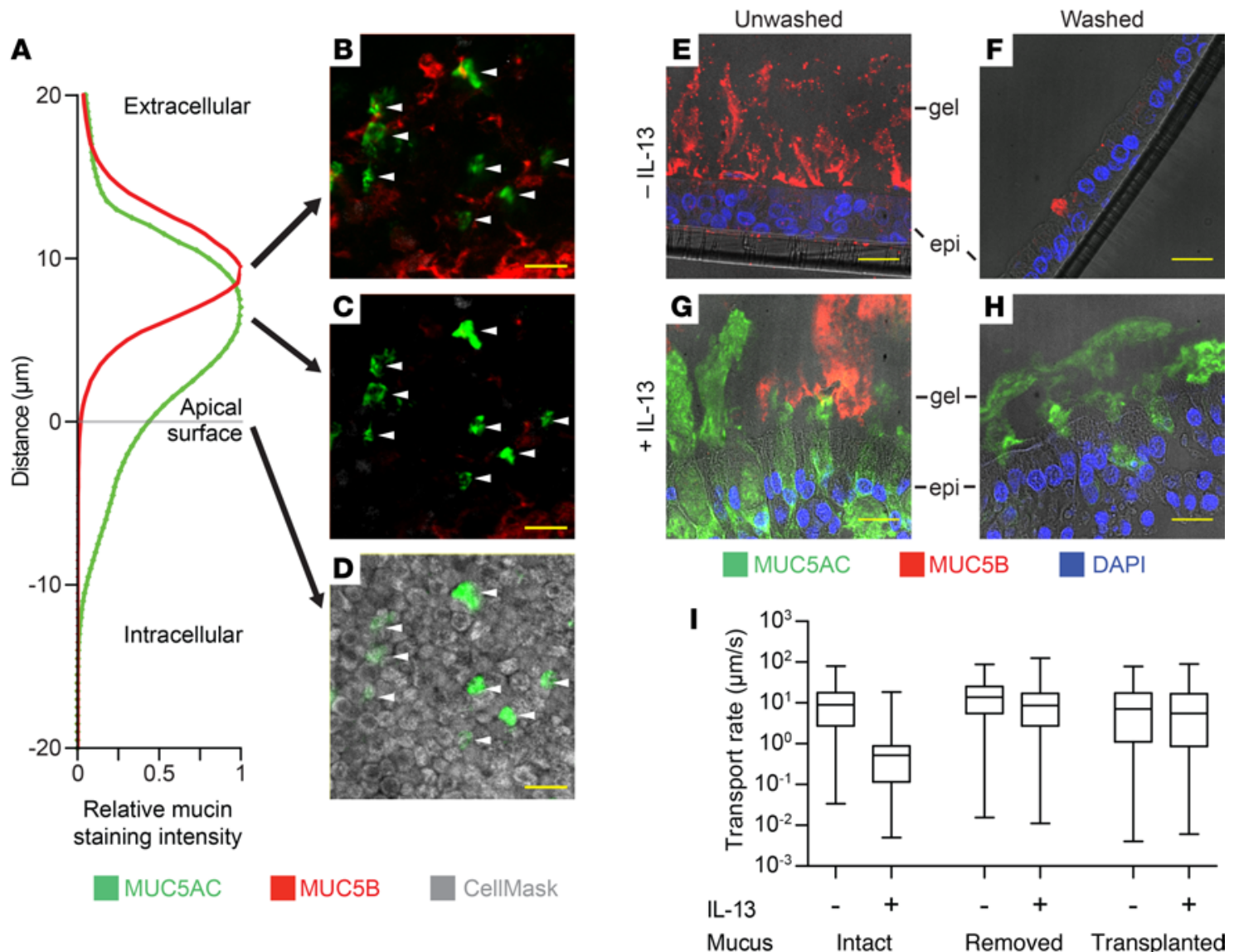

Figure 3. MUC5AC-rich gels are tethered to mucous cells in airway epithelium. (A) Distribution of mucin staining intensity as a function of distance from the apical surface of the epithelium. Values represent mean fluorescence intensities for MUC5AC and MUC5B staining from a Z-stack of images of a whole-mount preparation from 1 of 3 unwashed IL-13-stimulated HBE cell cultures. The apical surface of the epithelium was identified using a plasma membrane stain (CellMask). (B-D) Selected images from the Z-stack analyzed in A. Arrowheads indicate continuous regions of MUC5AC staining that extend from the surface of mucous cells (D) into the middle (C) and upper (B) portions of the mucus gel. Scale bars: $20 \mu \mathrm{m}$. (E-H) Immunohistochemical staining of sections from cultures immediately immersed in nonaqueous fixative (unwashed) or fixed after washing the apical surface to remove nonadherent mucus. Scale bars: $20 \mu \mathrm{m}$. (I) Distribution of microsphere velocities from unstimulated and IL-13-stimulated cultures before washing (mucus intact), after washing with solution containing DTT (mucus removed), and after addition of mucus from another culture of untreated HBE cells (transplanted). Velocities were determined from 881 to 5,563 microspheres in 3 fields of view from each of 3 cultures $(P<0.0001$ for all possible pairwise comparisons between the 6 conditions; Mann-Whitney $U$ test). Boxes extend from the 25 th to the 75 th percentiles, the line within the box indicates the median, and the whiskers represent minimum and maximum values.

Our results are consistent with the findings from this study and provide new evidence coupling previous histologic observations to new analyses of the mucin composition, structural organization, and function of pathologic mucus gels. MUC5AC and MUC5B are secreted from different cells, or from different granules within the same cell, and remain largely segregated extracellularly. Differences in the biophysical properties of MUC5AC- and MUC5B-containing domains could be attributable to intrinsic properties of the MUC5AC and MUC5B core proteins, although, unlike other mucins with transmembrane domains (sometimes called "tethered mucins"), MUC5AC and MUC5B are not integral membrane proteins. Other potentially important factors include posttranslational mucin modifications (e.g., glycosylation or disulfide cross-linking), hydration- and bicarbonate-mediated mucin expansion, non-mucin mucus constituents, or other differences in the secretory cell or the luminal environment.
Many mechanisms contribute to airway dysfunction in asthma. Some factors important in asthma exacerbations, including respiratory viruses and inflammatory cells, were absent in our model and may make independent contributions to impaired mucus transport. Nonetheless, our work shows that IL-13, a key mediator in many individuals with mild-to-moderate (16) and severe asthma (24), is sufficient to produce alterations in the mucus gel that closely resemble those observed in fatal asthma. These alterations result in a MUC5AC-rich gel that is tethered to epithelial mucous cells and markedly impairs mucociliary transport. MUC5AC tethering probably leads to progressive luminal accumulation of mucus and airway plugging. Hence, changes in mucus composition and organization are likely to be major contributors to airway obstruction, morbidity, and mortality in asthma.

\section{Methods}

Further information can be found in the Supplemental Methods and in Supplemental Figures 1-7. 
Statistics. Two-group comparisons were performed using the 2-tailed Student's $t$ test (normally distributed data) or the Mann-Whitney U test. A $P$ value of less than 0.05 was considered statistically significant.

Study approval. The UCSF Committee on Human Research approved the use of human airway sections and HBE cells. Written consent was not required, as materials were leftover clinical samples obtained from deidentified individuals.

\section{Author contributions}

LRB and DJE conceived the study and designed the experiments. LRB and LZ performed the experiments and acquired the data. LRB, LZ, WF, and DJE analyzed and interpreted the results. LRB and DJE wrote the manuscript.

\section{Acknowledgments}

This work was supported by NIH grant AIO77439 and by the Cystic Fibrosis Cell Models Core (funded by NIH grant DK072517 and Cystic Fibrosis Foundation grant DR613-CR11). Kurt Thorn and DeLaine Larsen (UCSF Imaging Center) and Carolin Boecking (UCSF) assisted with microscopy. Brian Button and Robert Williams (University of North Carolina) and Tim Acker (UCSF) provided advice on mucus solids measurements. We thank Dean Sheppard and Steven Rosen (UCSF) for their helpful suggestions.

Address correspondence to: David J. Erle, University of California San Francisco, Mail Code 2922, San Francisco, California 941432922, USA. Phone: 415.514.4370; E-mail: david.erle@ucsf.edu.
1. Croisant S. Epidemiology of asthma: prevalence and burden of disease. Adv Exp Med Biol. 2014;795:17-29.

2. Bateman JR, Pavia D, Sheahan NF, Agnew JE, Clarke SW. Impaired tracheobronchial clearance in patients with mild stable asthma. Thorax. 1983;38(6):463-467.

3. Messina MS, O'Riordan TG, Smaldone GC. Changes in mucociliary clearance during acute exacerbations of asthma. Am Rev Respir Dis. 1991;143(5 pt 1):993-997.

4. Messer JW, Peters GA, Bennett WA. Causes of death and pathologic findings in 304 cases of bronchial asthma. Dis Chest. 1960;38:616-624.

5. Aikawa T, Shimura S, Sasaki H, Ebina M, Takishima T. Marked goblet cell hyperplasia with mucus accumulation in the airways of patients who died of severe acute asthma attack. Chest. 1992;101(4):916-921.

6. Kuyper LM, et al. Characterization of airway plugging in fatal asthma. Am JMed. 2003;115(1):6-11.

7. Knowles MR, Boucher RC. Mucus clearance as a primary innate defense mechanism for mammalian airways. J Clin Invest. 2002;109(5):571-577.

8. Button B, et al. A periciliary brush promotes the lung health by separating the mucus layer from airway epithelia. Science. 2012;337(6097):937-941.
9. Hoegger MJ, et al. Cystic fibrosis. Impaired mucus detachment disrupts mucociliary transport in a piglet model of cystic fibrosis. Science. 2014;345(6198):818-822.

10. Thornton DJ, Rousseau K, McGuckin MA. Structure and function of the polymeric mucins in airways mucus. Annu Rev Physiol. 2008;70:459-486.

11. Groneberg DA, et al. Expression of MUC5AC and MUC5B mucins in normal and cystic fibrosis lung. Respir Med. 2002;96(2):81-86.

12. Roy MG, et al. Muc5b is required for airway defence. Nature. 2013;505(7483):412-416.

13. Evans CM, et al. The polymeric mucin Muc5ac is required for allergic airway hyperreactivity. Nat Commun. 2015;6:6281.

14. Kuperman DA, Huang X, Nguyenvu L, Holscher C, Brombacher F, Erle DJ. IL-4 receptor signaling in Clara cells is required for allergen-induced mucus production. JImmunol. 2005;175(6):3746-3752.

15. Kuperman DA, et al. Direct effects of interleukin-13 on epithelial cells cause airway hyperreactivity and mucus overproduction in asthma. Nat Med. 2002;8(8):885-889.

16. Woodruff PG, et al. T-helper type 2-driven inflammation defines major subphenotypes of asthma. Am J Respir Crit Care Med. 2009;180(5):388-395.

17. Kirkham S, Sheehan JK, Knight D, Richardson PS,
Thornton DJ. Heterogeneity of airways mucus: variations in the amounts and glycoforms of the major oligomeric mucins MUC5AC and MUC5B. Biochem J. 2002;361(pt 3):537-546.

18. Sheehan JK, Richardson PS, Fung DC, Howard $\mathrm{M}$, Thornton DJ. Analysis of respiratory mucus glycoproteins in asthma: a detailed study from a patient who died in status asthmaticus. Am J Respir Cell Mol Biol. 1995;13(6):748-756.

19. Zhu Y, et al. Munc13-2-/ baseline secretion defect reveals source of oligomeric mucins in mouse airways. J Physiol. 2008;586(7):1977-1992.

20. Hill DB, et al. A biophysical basis for mucus solids concentration as a candidate biomarker for airways disease. PLoS One. 2014;9(2):e87681.

21. Bermbach S, et al. Mechanisms of cilia-driven transport in the airways in the absence of mucus. Am J Respir Cell Mol Biol. 2014;51(1):56-67.

22. Rogers DF. Airway mucus hypersecretion in asthma: an undervalued pathology? Curr Opin Pharmacol. 2004;4(3):241-250.

23. Shimura S, Andoh Y, Haraguchi M, Shirato K. Continuity of airway goblet cells and intraluminal mucus in the airways of patients with bronchial asthma. Eur Respir J. 1996;9(7):1395-1401.

24. Corren J, et al. Lebrikizumab treatment in adults with asthma. $N$ Engl J Med. 2011;365(12):1088-1098. 